\title{
Type 1 Diabetes: Clinical and Experimental
}

\author{
Mikael Knipa and Riitta Veijolab \\ aHospital for Children and Adolescents, University of Helsinki, Helsinki, Finland \\ bepartment of Pediatrics, University of Oulu, Oulu, Finland
}

\begin{abstract}
Type 1 diabetes is a life-long disease associated with an increased risk for a variety of micro- and macrovascular complications. Encouraging information has been reported recently indicating that the frequency of diabetic nephropathy has decreased substantially over the last three decades of the twentieth century. This suggests that improved metabolic control and more aggressive treatment have borne fruit. Type 1 diabetes is, however, still associated with an at least 2 -fold increased mortality among children, adolescents and young adults. Accordingly there is a definite need of more knowledge both about the pathogenesis of the disease itself, its complications and effective treatment modalities.
\end{abstract}

\section{Mechanism of the year}

\section{The insulin A-chain epitope recognized by human $\mathbf{T}$ cells is posttranslationally modified}

Mannering SI, Harrison LC, Williamson NA, Morris JS, Thearle DJ, Jensen KP, Kay TW, Falk BA, Nepom GT, Purcell AW Autoimmunity and Transplantation Division, The Walter and Eliza Hall Institute of Medical Research,

Royal Melbourne Hospital, Parkville, Vic., Australia

mannering@wehi.edu.au

J Exp Med 2005;202:1191-1197

Background: The autoimmune process that destroys the insulin-producing pancreatic $\beta$ cells in type 1 diabetes targets insulin and its precursor, proinsulin. $T$ cells that recognize the proximal A-chain of human insulin were identified recently in the pancreatic lymph nodes of subjects who suffered from type 1 diabetes.

Methods: To investigate the specificity of proinsulin-specific T cells in type 1 diabetes, human $\mathrm{CD}^{+}$ T-cell clones to proinsulin were isolated from the blood of a donor affected by type 1 diabetes.

Results: The clones recognized a naturally processed, HLA-DR4-restricted epitope within the first 13 amino acids of the A-chain (A1-13) of human insulin. A vicinal disulfide bond between adjacent cysteine residues at A6 and A7 was critical for T-cell recognition. This disulfide bond did not alter binding of the peptide to HLA-DR4. CD4 ${ }^{+}$T-cell clones that recognized this epitope were isolated from an HLA-DR4-positive child with autoantibodies to insulin, and therefore, at increased risk for type 1 diabetes, but not from two non-diabetic HLA-DR4-positive subjects.

Conclusion: A novel posttranslational modification that is required for T-cell recognition of the insulin A-chain in type 1 diabetes was defined for the first time.

In parallel with our understanding of pre- and posttranslational modifications of hormone action, a series of different posttranslational modifications of antigens have now been described. One of the most intriguing is the deamidation of gluten peptides which is required for the binding of these peptides to the HLA-DQ2 molecule, a necessary step in the development of celiac disease [1]. Even more provoking is the observation that tissue transglutaminase, the critical autoantigen in celiac disease, catalyzes the deamidation of gluten peptides. Accordingly the posttranslational modification of dietary antigens is a crucial element in the pathogenesis of celiac disease. Here, Mannering et al. report that a disulfide bond between two adjacent cysteine residues ( $A 6$ and $A 7$ ) is essential for the recognition of the insulin A-chain peptide $A 1-13$ by $\mathrm{CD4}^{+} \mathrm{T}$ cells, although the disulfide bond formation did not affect the binding of the peptide to the HLA-DR4 molecule. This observation opens up interesting questions. Where does the posttranslational modification of the insulin peptide occur in man? Which enzyme regulates the disulfide bond formation? The posttranslational modification of 
the insulin peptide also fits into the hypothesis that bovine insulin could be the driving antigen in type 1 diabetes [2]. Most individuals are daily exposed to bovine insulin peptides by ingesting cow's milk and/or cow's milk derived products. The bovine insulin peptide A1-13 comprises the same cysteine residues at positions $A 6$ and $A 7$ as human insulin, and the intestinal tract would be an attractive location for disulfide bond formation. The insulin A-chain peptide is also of special interest, since Kent et al. [3] reported recently that $T$ cells isolated from the pancreatic draining lymph nodes of donors affected by type 1 diabetes recognized the insulin A1-15 epitope restricted by HLA-DR4. The present study reminds us that the immune system does not see native antigens but peptide motifs that can be quite dissimilar from the original protein. It raises the awareness that posttranslational modification of antigens may be an important element in the disease process of type 1 diabetes in parallel with other immune-mediated diseases.

\section{New paradigms}

\section{The 'accelerator hypothesis': relationship between weight, height, body mass index and age at diagnosis in a large cohort of 9,248 German and Austrian children with type 1 diabetes mellitus}

Knerr I, Wolf J, Reinehr T, Stachow R, Grabert M, Schober E, Rascher W, Holl RW, DPV Scientific Initiative of

Germany and Austria

University Children's Hospital, University of Erlangen-Nuremberg, Erlangen, Germany

ina.knerr@kinder.imed.uni-erlangen.de

Diabetologia 2005;48:2501-2504

Background: Previous studies have indicated that accelerated growth and overweight in childhood may be a risk factor for type 1 diabetes. This study set out to investigate whether either increased weight or BMI are associated with earlier presentation of type 1 diabetes mellitus in children.

Methods: Anthropometric measurements were examined in a large cohort of 9,248 children and adolescents who were diagnosed with type 1 diabetes over a 14-year period (1990-2003) in 116 pediatric clinics throughout Germany and Austria.

Results: The subjects were divided into four age groups (0-4.9, 5-9.9, 10-14.9 and 15-20 years). The three younger age groups were characterized by higher standard deviation scores (SDSs) for weight and BMI at diagnosis compared with the reference population $(\mathrm{p}<0.00001)$. This was true for both boys and girls. The BMI SDS and the weight SDS were higher in the youngest age group of affected children than in all other age groups $(\mathrm{p}<0.00001)$. BMI SDS at diagnosis gradually decreased with increasing age at disease manifestation ( $\mathrm{p}<0.0001)$. A continuous increase in the weight-SDS and the BMI-SDS was seen over the 14-year study period in the total cohort $(\mathrm{p}<0.0001)$, and in the two youngest age groups in particular. Male sex and year of disease manifestation had a significant influence on BMI SDS $(\mathrm{p}<0.0001)$ based on multivariate analysis, which also showed an inverse association between the patients' BMI SDS and age at diagnosis, with a mean annual decrease in BMI SDS of $-0.0248(\mathrm{p}<0.0001)$.

Conclusions: There was an association between a higher BMI and a younger age at the diagnosis of diabetes. Increased weight gain could accordingly be a risk factor for the early manifestation of type 1 diabetes.

This study confirms in a large series of children and adolescents with newly diagnosed type 1 diabetes that there is a negative association between BMI and age at diagnosis. This observation is not surprising, given that insulin resistance has been shown to be an independent risk factor for progression to type 1 diabetes in autoantibody-positive family members of affected children [4,5]. The data also show that there has been a steady increase in relative weight and BMI expressed in SDS among children and adolescents with newly diagnosed type 1 diabetes over the 14-year observation period. This finding supports the accelerator hypothesis and raises the issue of the relative contribution of increasing body mass to the conspicuous increase seen in the incidence rate of type 1 diabetes among children under the age of 15 years after World War II in most developed countries. Recently accumulated evidence, like the present data, suggests that interventions targeting lifestyle factors that enhance insulin sensitivity and favor $\beta$-cell rest, such as increased physical activity and a balanced 
energy intake, may have a role in population-based strategies for the prevention of both type 1 and type 2 diabetes. It remains to be defined which unique metabolic changes accompanying childhood obesity provide the link to the development of type 1 diabetes. These could include insulin, IGF-I, leptin and a variety of other hormones and metabolites.

\section{New hope}

\section{Incidence of end-stage renal disease in patients with type 1 diabetes}

Finne P, Reunanen A, Stenman S, Groop PH, Gronhagen-Riska C Finnish Registry for Kidney Diseases, Helsinki, Finland

patrik.finne@hus.fi

JAMA 2005;294:1782-1787

Background: One third of subjects with type 1 diabetes develop microalbuminuria, and $15-25 \%$ develop persistent proteinuria during the first 20 years of the disease. However, data on the long-term risk of developing end-stage renal disease (ESRD) is sparse. The objectives of the current study were to estimate the long-term risk of ESRD and death in patients with type 1 diabetes, and to study how age at diagnosis, time period of diagnosis and sex affect these risks.

Methods: All patients diagnosed with type 1 diabetes under the age of 30 years in Finland in 1965-1999 were identified from the Finnish Diabetes Register $(n=20,005)$. This cohort was observed from the diagnosis of type 1 diabetes until the manifestation of ESRD (dialysis or kidney transplantation), death or end of follow-up on December 31, 2001. Information on ESRD was obtained from the Finnish Registry of Kidney Diseases and data on deaths was acquired by database linkage with the Population Register Centre in Finland.

Results: The patients were observed for a median of 16.7 years (range 0-37.0). During 346,851 personyears, 632 patients developed ESRD and 1,417 died. The cumulative incidence of ESRD was $2.2 \%$ after 20 years and $7.8 \%$ after 30 years of follow-up. Patients diagnosed with type 1 diabetes before the age of 5 years had a lower risk of developing ESRD than others ( 3.3 vs. $8.4 \%$ after 30 years). The risk of ESRD was also reduced in patients who were diagnosed with diabetes in more recent years. There was no difference between the sexes in the risk of ESRD.

Conclusion: The risk for development of ESRD in patients with type 1 diabetes was observed to be substantially lower than previously reported indicating that the prognosis of type 1 diabetes has improved over the past four decades. Children diagnosed with diabetes below the age of 5 years had the most favorable prognosis with regard to ESRD.

This is the first population-based study estimating the long-term risk of developing end-stage renal disease (ESRD) in patients with type 1 diabetes, and the results, showing a more favorable prognosis than previously reported, really give new hope for both the patients and their doctors! The large number of patients included in the follow-up adds to the value of these results. It must be emphasized that this work was only possible by combining three nationwide Finnish registers, all of which are almost $100 \%$ complete. This study also represents a beautiful example of the benefits obtained from a homogeneous and accessible health care system. In Finland the incidence of type 1 diabetes is highest in the world among children and adolescents. Much emphasis has been put on diabetes care in Finland, and these results show that the efforts pay off. The cumulative incidence of ESRD within 30 years from the diagnosis of type 1 diabetes was $7.8 \%$, which is clearly lower than the previously reported $16 \%$. For the first time it was shown that children diagnosed under the age of 5 years have the most favorable prognosis in terms of ESRD but also in terms of death from any cause. Which factors could explain these observations? Is it just the fact that young children better adjust and more easily accept the disease and its treatment as an essential and permanent part of their lives? Or could there be genetic reasons behind the findings? The results of this excellent register-based study provoke such questions, which remain to be answered. One may speculate that a common genetic predisposition leads both to early manifestation of type 1 diabetes and a favorable renal prognosis. In that respect, the observation that the haptoglobin 1-1 phenotype may protect against the 
development of nephropathy in patients with diabetes and a normal serum creatinine concentration provides an interesting piece of information [6].

\section{New concerns}

\section{Mortality in childhood-onset type 1 diabetes: a population-based study}

Dahlquist G, Kallen B

Department of Clinical Sciences, Pediatrics, Umea University, Umea, Sweden

gisela.dahlquist@pediatri.umu.se

Diabetes Care 2005;28:2384-2387

Background: Type 1 diabetes is associated with considerable excess mortality at all ages. Most of the increased mortality is known to be due to long-term complications. However, an increased death rate has also been reported among young patients without signs of long-term complications. This study describes age- and sex-specific mortality in a cohort of young subjects with type 1 diabetes, and analyzes the causes of death with special focus on suicides, accidents and unexplained deaths.

Methods: Mortality of children diagnosed with type 1 diabetes at the age of $0-14$ years during the period between July 1, 1977 and December 31, $2000(\mathrm{n}=10,200)$ was studied by linking the populationbased Swedish Childhood Diabetes Register with the Swedish Cause of Death Register. To calculate age- and sex-specific standardized mortality rates (SMR), data from the official Swedish population register was used. Neonatal deaths were excluded. To analyze whether cases with diabetes have an excess death rate for specific diagnoses, each case was compared with 5 non-diabetic control subjects matched by age, sex and year of death. Death certificates were collected for all these subjects and in the case of unclear diagnosis, hospital records and/or autopsy reports were obtained.

Results: A total of 78 young patients with diabetes ( 49 males and 29 females) died during the observation period of 81,600 person-years. Mean age at death was 15.2 years (range 1.2-27.3) and mean duration of diabetes 8.2 years (0-20.7). The mean SMR was 2.15 (95\% CI 1.70-2.68) being slightly higher for females (2.65 vs. 1.93) and peaking up to 4.66 in patients diagnosed with diabetes before the age of 2 years. Twenty-three deaths were clearly related to diabetes, 20 of these being diabetic ketoacidosis. Twenty deaths were uncertainly related to diabetes, 17 of these being unexplained deaths in bed at home. Thirty-three deaths were not directly related to diabetes, and 2 deaths remained unclear (death abroad, no diagnosis). There was no statistically significant difference in traffic accidents or obvious suicides between the subjects with diabetes and controls. Unexplained death in bed at home was found in only 2 control subjects.

Conclusion: There is still a significant excess mortality in young patients with type 1 diabetes, even in a well-developed health care system. Traffic accidents or suicide are not clearly increased among young diabetic subjects, whereas a large proportion of unexplained deaths in bed is evident and should be further investigated.

This is another example of the usefulness of population-based registers - now telling us sad news about the increased mortality rate still present among young individuals with type 1 diabetes. The age- and sex-specific standardized mortality rate (SMR) was more than 4 for children diagnosed before the age 2 years, and about 2 among those diagnosed between 3 and 15 years of age. The most common cause for death $(29.5 \%)$ was related to diabetes: 6 deaths at diagnosis $(5$ were $<4$ years of age), 14 deaths because of ketoacidosis ( 4 treated in hospital and 10 subjects living alone and found at home), 1 alcohol intoxication with suspected hypoglycemia, and 2 acute coronary infarctions. The striking result was the high proportion of unexplained deaths in bed: $22 \%$ of all deaths among the diabetic cases compared to only $0.6 \%$ among the controls. As the authors conclude, this observation needs further investigation since the reasons for these sudden deaths during sleep are open at the moment. Hypoglycemia could lead to death particularly if associated with dysregulation in counter-regulatory hormones. Hypoglycemia has also been reported to induce cardiac arrhythmia eventually leading to death in otherwise healthy young individuals. Another conspicuous result is the relatively large number of deaths from diabetic ketoacidosis, together with the fact that 
most of these cases were patients who lived alone and died at home. Laing et al. [7] have recently studied psychosocial and socioeconomic risk factors for premature death in young people with type 1 diabetes in a nested case-control setting in the UK. The cases had type 1 diabetes and died before the age of 40 years. A total of 98 deaths were recorded and 51 of these were classified as acute events including sudden episodes of diabetes such as hypoglycemia and coma, as well as deaths from accidents and violence. Living alone (odds ratio (OR) 4.4), past drug abuse (OR 5.7) and previous psychiatric referral (OR 4.6) were associated with deaths from acute events. What can be done to prevent excess mortality among young patients with diabetes? Clearly, one option is early diagnosis of diabetes in very young children which will prevent deaths associated with severe diabetic ketoacidosis at onset. Psychosocial factors should be taken into account on a regular basis in the treatment, and young individuals with known risk factors for sudden death like living alone, drug abuse, psychiatric referral, should be given particular support.

\title{
Spectrum and prevalence of atherogenic risk factors in 27,358 children, adolescents, and young adults with type 1 diabetes: cross-sectional data from the German diabetes documentation and quality management system (DPV)
}

\author{
Schwab KO, Doerfer J, Hecker W, Grulich-Henn J, Wiemann D, Kordonouri O, Beyer P, Holl RW, DPV Initiative of the \\ German Working Group for Pediatric Diabetology \\ Department of Pediatrics and Adolescence Medicine, Freiburg University Hospital, Freiburg, Germany \\ schwab@kikli.ukl.uni-freiburg.de \\ Diabetes Care 2006;29:218-225
}

Background: Type 1 diabetes is associated with increased cardiovascular morbidity and mortality in young and middle-aged adults. This data analysis aimed at ascertaining the type and prevalence rate as well as age and sex distribution of cardiovascular risk factors in patients with type 1 diabetes up to the age of 26 years.

Methods: 27,358 patients were assessed for cardiovascular risk factors such as obesity, hypertension, dyslipidemia, poor glycemic control, and smoking. The patients were categorized into three age groups, i.e. prepubertal ( $<12$ years of age), pubertal (12-16 years), or adult (17-26 years).

Results: At least one cardiovascular risk factor was present in more than half of the patients in each group. Two risk factors were observed in $6.2-21.7 \%$ of the patients, the lowest prevalence seen among prepubertal patients and the highest among adult patients. Three or four risk factors were detected in $0.5-4.7 \%$ of the patients depending on age. Increased values of HbA1c, total cholesterol, and BMI were seen most frequently. Hypertension, smoking, and decreased HDL cholesterol were observed more frequently in males, while females had increased BMI, total cholesterol, and LDL cholesterol more often than males. Only $0.4 \%$ of the patients were treated for dyslipidemia, although $28.6 \%$ of them had signs of dyslipidemia. Close to $2 \%(2.1 \%)$ were treated with antihypertensive medication, while about four times more $(8.1 \%)$ had hypertension.

Conclusions: The prevalence of cardiovascular risk factors increased along with older age in this cohort comprising children, adolescents and young adults with type 1 diabetes. Significant gender differences were observed in the majority of risk factors. Despite a high prevalence of risk factors, only a small minority of the patients were treated with antihypertensive or lipid-lowering treatment. Identification and early prevention of future cardiovascular disease need to be implemented already in childhood among subjects affected by type 1 diabetes.

This is an impressive cohort of young patients with type 1 diabetes, although data on all risk factors were not available from every subject. The frequency of two or more risk factors increased clearly with increasing age, but already more than $5 \%$ of those younger than 12 years of age had two or more risk factors. This indicates that screening for cardiovascular risk factors should be initiated at the latest in early puberty. Most of the risk factors are modifiable, and hence screening is only the first step which should be followed by appropriate intervention measures. Improved metabolic control and weight control are important goals when aiming at reducing later cardiovascular morbidity and mortality. Increased physical activity and a healthy diet have a beneficial effect on most cardiovascular risk factors, and are accordingly primary intervention modalities. If the goals cannot be achieved by lifestyle modifications, drug therapy should be considered for those risk factors that can 
be successfully and relatively safely modified by pharmacological means. This applies to hypertension and dyslipidemia, in particular. The gender differences in the risk factor profile suggest that there may be a need to establish gender-specific intervention programs, e.g. weight-controlling measures appear to be more important among females, whereas interventions aimed at stopping smoking seem to be more pertinent among males.

\title{
Frequency and timing of severe hypoglycemia affects spatial memory in children with type 1 diabetes
}

\author{
Hershey T, Perantie DC, Warren SL, Zimmerman EC, Sadler M, White NH \\ Department of Psychiatry, Washington University School of Medicine, St. Louis, Mo., USA \\ tammy@wustl.edu \\ Diabetes Care 2005;28:2372-2377
}

Background: Repeated severe hypoglycemia has been reported to reduce long-term spatial memory. This study aimed at determining whether the age at which severe hypoglycemia occurs modulates its impact on long-term spatial memory.

Methods: Subjects from three previous studies were combined to obtain a cohort comprising children aged 6-18 years with diabetes $(n=103)$ and non-diabetic control subjects $(n=60)$. Each study had tested short ( $5 \mathrm{~s}$ )- and long (60 s)-delay spatial memory with the spatial delay response task and evaluated its association with severe hypoglycemia. The subjects were categorized as having $0,1-2$, or $\geq 3$ severe hypoglycemic episodes, and as having their first severe hypoglycemic episode before or after the age of 5 years. Data on hypoglycemic events was collected retrospectively through parental report and chart review. All available HbA1c values from diagnosis to testing were collected from medical charts and mean $\mathrm{HbA1c}$ was calculated. Exposure to chronic hyperglycemia was estimated by multiplying the proportion of HbA1c values $>9.0 \%$ by the duration of type 1 diabetes for each individual.

Results: Repeated severe hypoglycemia (at least 3 episodes) was observed to be associated with reduced long-delay spatial memory performance on a spatial delayed response test, particularly when severe hypoglycemic events had started before the age of 5 years. Age at onset of diabetes, mean HbA1c and the estimated chronic hyperglycemia exposure were not associated with performance.

Conclusion: Both high frequency and early exposure to severe hypoglycemia have negative effects on spatial long-term memory performance in children with type 1 diabetes.

Long-term cognitive effects of severe hypoglycemia have been debated in children affected by type 1 diabetes. We know that young children with type 1 diabetes are at greatest risk of severe hypoglycemic events. Several studies have reported adverse effects of severe hypoglycemia on a variety of cognitive abilities, such as memory, attention and verbal IQ, whereas others have failed to demonstrate a systematic association between severe hypoglycemia and compromised intellectual function. The most consistent finding has been, however, that diabetes presentation at young age predicts poorer cognitive function, pointing to the possibility that severe hypoglycemia occurring early in development is more harmful to the cognitive function than severe hypoglycemia later in development. However, few studies have actually evaluated whether the age at which severe hypoglycemia occurs is important in determining the cognitive outcome. In the present paper this question was addressed by combining three earlier series of patients and controls who had all been tested for spatial memory performance in a similar way. The results from two of these patient series have been published earlier and therefore, it was not surprising that the authors 'confirmed' their earlier result that repeated severe hypoglycemia reduces long-term spatial memory performance. The study has several limitations, however. Data on hypoglycemias were retrospectively collected as well as the $\mathrm{HbA1C}$ values. Sample size in the subgroup analyses was modest. Furthermore, subjects with a high frequency of hypoglycemic episodes had a longer diabetes duration than those with fewer episodes, which may cause bias. The authors also admitted that the relevance of their findings to everyday aspects of learning and memory are not known. In contrast, a paper from an Australian group published last year found no clear evidence that episodes of severe hypoglycemia, even those occurring very early in childhood, resulted in cognitive dysfunction or memory difficulties [8]. That study recorded prospectively all hypoglycemic events that resulted in seizure or coma at each 3-month clinic visit since the diagnosis of diabetes. Diabetic children with a documented history of severe hypoglycemic events $(n=41)$ and diabetic children without such episodes $(n=43)$ were compared. 
The tests used in the evaluation of learning, memory, intelligence and behavior were done at the age of 6-15 years and were partly different than those used in the study by Hershey et al., making direct comparison of the two papers difficult. Clearly, long-term prospective studies with larger sample sizes and using validated measures are needed to define the true and clinically meaningful consequences of severe childhood hypoglycemia in terms of later cognitive function of the diabetic child and adult. A recent experimental study indicated that the administration of pyruvate after severe hypoglycemia reduced neuronal death and cognitive function [9], raising the issue of the potential therapeutic impact of pyruvate in children with severe hypoglycemia.

\title{
Concepts revised
}

\section{Patterns of metabolic progression to type 1 diabetes in the diabetes prevention trial-type 1}

\author{
Sosenko JM, Palmer JP, Greenbaum CJ, Mahon J, Cowie C, Krischer JP, Chase HP, White NH, Buckingham B, \\ Herold KC, Cuthbertson D, Skyler JS \\ Division of Endocrinology, University of Miami, Miami, Fla., USA \\ jsosenko@med.miami.edu
}

Diabetes Care 2006;29:643-649

Background: There is limited information available on the pattern of metabolic deterioration before the diagnosis of type 1 diabetes. This study utilized data from the Diabetes Prevention Trial-Type 1 (DPT-1) to characterize the metabolic progression to type 1 diabetes over a period of approximately 2.5 years before its diagnosis.

Methods: 54 DPT-1 participants (22 in the parenteral trial and 32 in the oral trial) were observed. Oral glucose tolerance tests (OGTTs) were performed in all subjects at 6-month intervals from approximately 30 to 6 months before diagnosis. An OGTT was also performed in the vast majority at disease presentation. Changes in OGTT glucose and C-peptide indexes from 30 to 6 months before diagnosis were assessed by calculating slopes of the indexes for each individual over that time period. Changes from 6 months before diagnosis to diagnosis were examined by paired comparisons of the OGTT metabolic indexes between the time points.

Results: There was a gradual increase in glucose levels from 30 to 6 months before diagnosis in both the parenteral and oral groups ( $\mathrm{p}<0.001$ for all indexes). A decrease was observed in the area under the curve (AUC) C-peptide $(\mathrm{p}<0.05)$ and AUC C-peptide-to-AUC glucose ratio $(\mathrm{p}<0.001)$ in the oral group, while peak C-peptide-to-2-h glucose ratio values decreased in both groups ( $\mathrm{p}<0.001)$. In participants who also had OGTTs at diagnosis, there was a decrease in AUC C-peptide (parenteral group, $\mathrm{p}<0.05$ ) and peak C-peptide (oral group, $\mathrm{p}<0.05$ ) over the last 6 months before diagnosis, whereas stimulated C-peptide-to-glucose ratio values decreased in both groups $(\mathrm{p}<0.001)$. Conversely, an increase was seen in fasting C-peptide levels in both groups (oral group, $\mathrm{p}<0.01$ ). No changes were observed in fasting C-peptide-to-fasting glucose ratio values over the 30 -month observation period.

Conclusions: Fasting C-peptide levels are maintained during the prediabetic disease process up to the diagnosis of type 1 diabetes. In contrast, stimulated C-peptide levels slowly decline in a considerable proportion of individuals who progress to type 1 diabetes as a sign of deteriorating glucose tolerance.

This is an interesting study on the metabolic course of preclinical type 1 diabetes based on participants in the Diabetes Prevention Type 1 (DPT-1) Trial who developed overt disease during prospective observation. The data show that $85 \%$ of the patients (40/47) were diagnosed with type 1 diabetes based on a diabetic OGTT, and only 7 presented with clinical symptoms when the subjects had repeated OGTTs with an interval of 6 months in the preclinical period. This observation suggests that the diagnosis of diabetes can be made earlier in an asymptomatic phase, when risk individuals are monitored for their glucose tolerance on a regular basis. The benefits of early diagnosis comprise better preserved $\beta$-cell function, milder metabolic decompensation including avoidance of diabetic ketoacidosis and decreased initial hospitalization rate [10]. The observation that fasting serum C-peptide concentrations remained stable during the observation period starting approximately 30 months 
before the diagnosis, whereas there was a gradual decrease in stimulated serum C-peptide concentrations, particularly when these were related to concomitant blood glucose levels, suggests that glucose-stimulated insulin secretion is a more sensitive marker of impaired $\beta$-cell function. This is not a surprising finding, given that an impaired insulin response to intravenous glucose has previously been shown to be the most sensitive indicator of $\beta$-cell dysfunction in preclinical type 1 diabetes [11]. This paper shows that more than one third of the subjects had signs of glucose tolerance abnormalities, such as impaired fasting glucose or impaired glucose tolerance, already at the time of the first OGTT about 3.5 years before the diagnosis of type 1 diabetes, illustrating that individuals may have considerable metabolic abnormalities and still not progress to diabetes for years.

\title{
The relationship between microalbuminuria and glomerular filtration rate in young type 1 diabetic subjects: the Oxford Regional Prospective Study
}

\author{
Amin R, Turner C, van Aken S, Bahu TK, Watts A, Lindsell DR, Dalton RN, Dunger DB \\ University Department of Paediatrics, Addenbrookes Hospital, Cambridge, UK \\ dbd25@cam.ac.uk
}

Kidney Int 2005;68:1740-1749

Background: Experimental studies have indicated that glomerular hypertension and hyperfiltration mediate progressive renal damage. In addition, hyperglycemia is known to promote glomerular hyperfiltration. The aim of this study was to examine the association between glomerular filtration rate (GFR) at 5 years' duration of type 1 diabetes and development of microalbuminuria in a prospective cohort of affected children.

Methods: 308 children participating in the Oxford Regional Prospective Study were followed from the diagnosis of type 1 diabetes with annual assessments of HbA1c and three urine samples for albumin:creatinine ratio (ACR), and measurement of GFR after 5 years' duration of diabetes. The mean age of the subjects was 9.8 years (range $0.4-15.9$ ) at diagnosis and the median duration of diabetes 10.9 years (range 6.0-17.8) at the end of follow-up. Microalbuminuria (MA) was defined as an ACR $>3.5 \mathrm{mg} / \mathrm{mmol}$ in males and $>4.0 \mathrm{mg} / \mathrm{mmol}$ in females, but $<35 \mathrm{mg} / \mathrm{mmol}$ in two of the three consecutive early morning urine samples.

Results: Most subjects $(\mathrm{n}=243,78.8 \%$ ) remained normoalbuminuric (MA-) throughout the study. At 5 years, $35(11.4 \%)$ subjects were microalbuminuric (MA+) and another 30 (9.7\%) were normoalbuminuric but developed MA+ during the subsequent follow-up, within a median of 3.3 years (future $\mathrm{MA}+$ ). GFR was higher in the future MA+ group compared to the MA+ and MA- groups (167 vs. 134 vs. $139 \mathrm{ml} / \mathrm{min} / 1.73 \mathrm{~m}^{2} ; \mathrm{p}<0.002$ ). In addition, the prevalence of glomerular hyperfiltration (GFR $>125 \mathrm{ml} / \mathrm{min} / 1.73 \mathrm{~m}^{2}$ ) was greater (97 vs. 57 vs. $64 \%$, future MA+, MA+, MA-; p = 0.006) and HbA1c levels were higher $(11.4$ vs. 10.8 vs. $9.7 \% ; \mathrm{p}<0.001)$ in the future MA+ group. Comparing subjects with and without hyperfiltration at a diabetes duration of 5 years, no difference in annual ACR levels existed prior to the first GFR measurement, whereas after this time the ACR levels were significantly higher in those with hyperfiltration, independent of age and HbA1c levels. The risk of developing MA between a diabetes duration of 5 and 10 years was associated with poor glycemic control and higher GFR at 5 years.

Conclusion: Glomerular hyperfiltration is predictive of microalbuminuria independent of metabolic control. This suggests that factors other than poor glycemic control are related to the development of hyperfiltration and diabetic nephropathy. Early medical intervention to reduce GFR may benefit poorly controlled subjects who hyperfiltrate even before the onset of microalbuminuria.

This is an important population-based, natural history study on the early signs of diabetic nephropathy in children with type 1 diabetes. The subjects took part in a longitudinal follow-up starting from the diagnosis of type 1 diabetes and were annually sampled to measure urine ACR. GFR was determined in all children after a diabetes duration of 5 years, and the annual assessment of urine ACR continued thereafter. It is clearly shown that glomerular hyperfiltration is present prior to increased urine ACR levels and is predictive of microalbuminuria (MA). The positive predictive value of hyperfiltration for the development of MA was only $15.7 \%$, however. The negative predictive value was as high as $\mathbf{9 8 . 9 \%}$, meaning that in the absence of hyperfiltration the risk for MA was minimal even in the presence of high $\mathrm{HbA1c}$. Therefore, glomerular hyperfiltration can be considered as a prerequisite for nephropathy. The reasons leading to hyperfiltration are not known and still require further 
research. The authors speculate whether pubertal hormones and growth hormone may be involved, but the levels of these hormones were not reported. Puberty was assumed to start at the age of 11 years (no Tanner classification was available), and therefore the results showing an association between puberty and glomerular hyperfiltration must be taken with caution. Since glomerular hyperfiltration was strongly predictive of the development of MA, the authors suggest that early intervention with medical therapy to reduce GFR should be considered in pubertal subjects who hyperfiltrate. Angiotensin-converting enzyme inhibitors and angiotensin receptor blockers could be highly effective in reducing glomerular hyperfiltration. However, randomized controlled trials testing these drugs as an early intervention to prevent diabetic nephropathy are needed. The proportion of hyperfiltrating adolescents with type 1 diabetes was as high as $67.8 \%$ of all subjects in this study, implying that a substantial proportion of young subjects would be exposed to these drugs if glomerular hyperfiltration was used as the only criteria for treatment. We know that only approximately $25 \%$ of subjects with type 1 diabetes will eventually develop nephropathy. Therefore, it seems that the group of adolescents really at risk of MA should be more clearly defined, maybe in terms of genetic markers, family history, and smoking before going for large-scale drug trials aimed at prevention of diabetic nephropathy.

\section{Important for clinical practice}

\section{Age, metabolic control and type of insulin regime influences health-related quality of life in children and adolescents with type 1 diabetes mellitus}

Wagner VM, Muller-Godeffroy E, Hager S, Thyen U

Klinik für Kinder- und Jugendmedizin, Universitätsklinikum Schleswig-Holstein, Lübeck, Germany

wagnerv@paedia.ukl.mu-luebeck.de

Eur J Pediatr 2005;164:491-496

Background: In the treatment of type 1 diabetes, enhancing quality of life (QOL) and well-being is as important as good metabolic control and prevention of secondary morbidity. This study evaluated health-related quality of life (HRQOL) in children (aged 8-12 years) and adolescents (aged 13-16 years) with type 1 diabetes and compared their results with healthy peers and children with other chronic conditions.

Methods: A total of 68 children and adolescents with type 1 diabetes for at least 5 months and regularly followed up at an interval of 4-8 weeks in the pediatric outpatient clinic of the University Hospital in Lübeck completed a generic German KINDL-R questionnaire, which has altogether 24 items reflecting six subscales (physical and psychological well-being, self-esteem, family, friends and school). In addition, this instrument has a module with 6 items assessing HRQOL related to a chronic condition. The items are statements, and the subject is asked to give a response on a scale ranging from 1 (never) to 5 (always). Higher scores indicated better HRQOL. Reference data from 1,502 schoolchildren was used for the six generic subscales and from 1,050 children with other chronic conditions for the additional scale. HbA1c levels were analyzed by HPLC, and good metabolic control was defined as a HbA1c $<8.0 \%$. Severe hypoglycemic events were recorded as well as data on height and weight and number of daily injections. Intensified therapy was defined as three or more daily injections.

Results: No differences were found in the total HRQOL sum scores between children and adolescents with type 1 diabetes and healthy peers. When analyzing subscales, children with type 1 diabetes reported significantly higher scores for school $(\mathrm{p}=0.007)$ and adolescents with type 1 diabetes for psychological well-being $(\mathrm{p}=0.012)$ compared to healthy controls. The younger age group of children with type 1 diabetes reported higher overall scores than the older age group, the difference being due to higher scores for school $(\mathrm{p}=0.001)$. Children and adolescents with good metabolic control reported better physical well-being $(\mathrm{p}=0.012)$ and those on intensified insulin treatment higher levels of HRQOL in the subscales friends and school $(\mathrm{p}=0.010)$. The additional module concerning the impact of chronic illness revealed that children and adolescents with type 1 diabetes had significantly higher HRQOL scores compared to the group of peers with other chronic diseases. 
Conclusion: Children and adolescents with type 1 diabetes who are followed up in a pediatric department with excellent resources and special expertise in diabetes management report good HRQOL. Younger age, lower HbA1c and intensified insulin therapy in subjects with type 1 diabetes were associated with significantly better HRQOL on different subscales. These results provide evidence that the time, staff, and cost required to treat children and adolescents with type 1 diabetes may result in good outcomes and be cost-saving in the long term.

\begin{abstract}
Although it is widely accepted that psychosocial care is an important element in good clinical practice when treating children and adolescents with type 1 diabetes, it is evident that most pediatric clinics do not use any structured instruments to assess psychosocial and emotional well-being of affected children and their families. These investigators were lucky to have a validated measure, KINDL-R for generic health-related quality of life (HRQOL), originally created for German children. In addition, they were testing this instrument in a remarkably well-resourced unit for the treatment of children with type 1 diabetes. Would it be the same if we applied the KINDL-R questionnaire in different countries and would we get similarly good results in our own clinics probably less resourced than the clinic in Lübeck? In any case, it is also important to discuss whether a selection bias might have hampered the results in this and other questionnaire studies, since subjects doing worst are more likely to refuse to participate. A separate questionnaire for the staff at the diabetes clinic would partly resolve this problem, and patients really needing extra support could be more easily identified. Another concern is the fact that we should have a disease-specific measure which would be especially useful when evaluating the effect of diabetes on everyday life, or comparing treatment between centralized and regional centers or within the same center at regular intervals. A very recent paper has now validated a short form of the Diabetes Quality of Life for Youth (DQOLY-SF) questionnaire in a large sample of children and adolescents aged 10-18 years from various countries across Europe, Asia and North America [12]. There is a hope that this measure, which is a shortened version from the original 52 -item questionnaire, will be clinically acceptable and applicable in the assessment of QOL in young individuals with type 1 diabetes.
\end{abstract}

\title{
Clinical trials, new treatments
}

\section{Use of inhaled insulin in a basal/bolus insulin regimen in type 1 diabetic subjects: a 6-month, randomized, comparative trial}

\author{
Skyler JS, Weinstock RS, Raskin P, Yale JF, Barrett E, Gerich JE, Gerstein HC, Inhaled Insulin Phase III \\ Type 1 Diabetes Study Group \\ University of Miami, School of Medicine, Miami, Fla., USA \\ jskyler@miami.edu
}

Diabetes Care 2005;28:1630-1635

Background: Patients do not always accept the basal/bolus insulin therapy for type 1 diabetes, although its benefits in terms of metabolic control have been clearly shown. This study set out to investigate whether a basal/bolus insulin regimen involving rapid-acting, dry powder, inhaled insulin could provide glycemic control comparable with a basal/bolus subcutaneous regimen.

Methods: 328 patients with type 1 diabetes aged 12-65 years were treated with subcutaneous NPH insulin twice daily and were randomized to receive either premeal inhaled insulin $(\mathrm{n}=163)$ or subcutaneous regular insulin $(\mathrm{n}=165)$ for 6 months.

Results: There was a comparable decrease from baseline in mean HbA1c values in the inhaled and subcutaneous insulin groups $(-0.3$ and $-0.1 \%$, respectively; adjusted difference $-0.16 \%$ [CI -0.34 to $0.01])$. A similar proportion of the patients achieved a $\mathrm{HbA} 1 \mathrm{c}$ value $<7 \%$. There were no differences in 2-hour postprandial glucose reductions between the groups, but fasting plasma glucose concentrations decreased more in the inhaled than in the subcutaneous insulin group (adjusted difference $-2.19 \mathrm{mmol} / \mathrm{l}$ [CI -3.19 to -1.20]). The overall hypoglycemia rate was lower but the severe hypoglycemia rate higher in the inhaled insulin group. The overall hypoglycemia rate (episodes/patient-month) was 9.3 (inhaled) vs. 9.9 (subcutaneous) (risk ratio [RR] 0.94 [CI 0.91-0.97]), and the severe hypoglycemia rate 
(episodes/100 patient-months) was 6.5 vs. 3.3 (RR 2.00 [CI 1.28-3.12]). The participants in the inhaled insulin group had increased insulin antibody binding in the peripheral circulation without associated clinical manifestations. Pulmonary function between the groups remained comparable with the exception that the patients in the inhaled insulin group experienced a decrease in carbon monoxide diffusing capacity without any clinical correlates.

Conclusions: The authors conclude that since there are patients who are unwilling or unable to use preprandial insulin injections, inhaled insulin may provide a tempting alternative for the bolus therapy on the management of type 1 diabetes.

Inhaled insulin could be an attractive option to rapid-acting meal insulins in specific categories of patients with type 1 diabetes, such as young children and old people. This study shows that inhaled insulin can replace subcutaneous bolus insulin without any adverse effects in terms of metabolic control. The observed decrease in fasting blood glucose in the patients treated with inhaled insulin is intriguing, and the mechanism behind this phenomenon remains unsolved. The higher frequency of severe hypoglycemia is an issue of concern, especially when considering the patient groups that would potentially benefit most from inhaled insulin. Similarly the increased formation of insulin antibodies induced by inhaled insulin represents an adverse effect, and indicates that inhaled insulin should not be used in women of fertile age, since high maternal insulin antibodies leads to transplacental transfer of insulin-insulin antibody complexes resulting in increased fetal hyperinsulinemia and macrosomia. The patients in the present study were treated with inhaled insulin for 6 months, which is still a very short period of time, when considering that the youngest patients randomized will remain on insulin therapy for 60 years or longer. The safety of long-term use of inhaled insulin remains to be assessed. It is surprising that this paper did not address at all patient satisfaction with inhaled insulin and does not provide any information on how many of the patients treated with inhaled insulin would have preferred to stay on that treatment at the end of the study. A very recent study reported that inhalation of human insulin results in small, consistent changes in pulmonary function tests and increased insulin antibody levels [13].

\section{Pharmacokinetics, prandial glucose control, and safety of insulin glulisine in children and adolescents with type 1 diabetes}

Danne $\mathrm{T}$, Becker RH, Heise $\mathrm{T}$, Bittner $\mathrm{C}$, Frick AD, Rave $\mathrm{K}$

Kinderkrankenhaus auf der Bult, Diabetes-Zentrum für Kinder und Jugendliche, Hannover, Germany

danne@hka.de

Diabetes Care 2005;28:2100-2105

Background: Insulin glulisine is a recombinant insulin analog designed to provide the same overall glucodynamic effect as regular human insulin (RHI) but a faster onset and shorter duration of action. These characteristics have been obtained by replacing asparagine with lysine at position 3 and lysine with glutamic acid at position 29 on the B-chain of the human insulin molecule. This study was conducted to investigate the pharmacokinetics, postprandial blood glucose profile, and safety of insulin glulisine in comparison with RHI in children and adolescents with type 1 diabetes.

Methods: A total of 20 young subjects with type 1 diabetes (10 children aged 5-11 years and 10 adolescents aged 12-17 years) were enrolled in a randomized, double-blind, cross-over study. Each subject received a single dose of $0.15 \mathrm{IU} / \mathrm{kg}$ of either insulin glulisine or RHI subcutaneously 2 min before a standardized liquid meal on two separate mornings after an overnight fast and a variable intravenous infusion of RHI to maintain blood glucose level at $5.6-8.9 \mathrm{mmol} / \mathrm{l}$ before the experiment.

Results: Maximum serum insulin concentrations were higher (58 vs. $33 \mathrm{mU} / \mathrm{l} ; \mathrm{p}<0.05$ ) and initial serum insulin concentration assessed by the area under the curve (AUC) for insulin during the first $2 \mathrm{~h}$ after the injection (INS $\left.\mathrm{AUC}_{0-2} \mathrm{~h}\right)$ was higher for insulin glulisine than RHI $(\mathrm{p}<0.05)$. Both the time to maximum insulin concentration (54 vs. $66 \mathrm{~min}$ ) and mean residence time in the systemic circulation ( 88 vs. $137 \mathrm{~min} ; \mathrm{p}<0.05)$ were shorter for insulin glulisine. Postprandial glucose levels were significantly lower after insulin glulisine vs. RHI assessed by blood glucose AUCs, maximum blood glucose and maximum change in the blood glucose (all $\mathrm{p}<0.05$ ). When comparing children and adolescents the pharmacokinetics of insulin glulisine was similar, whereas for RHI adolescents showed $64 \%$ higher concentrations than children. Insulin glulisine was also found to be safe and well tolerated in young patients. 
Conclusion: Similar rapid-acting properties of insulin glulisine that have previously been observed in adults were also demonstrated in children and adolescents with type 1 diabetes.

These results indicate that insulin glulisine is a promising new insulin analog also for children with pharmacokinetic properties which are very good for a mealtime insulin and clearly better than $\mathrm{RHI}$. Of course one has to take into account which long-acting insulin has been selected and how many daily injections are tolerated by the child and the family when choosing a mealtime insulin for a very young patient - no standard solution can be given for such scenarios. Later studies comparing directly insulin glulisine with insulin aspart and insulin lispro will probably tell us more about the similarities and/or differences between these three rapid-acting insulin analogs. At the end of the day, it will be to the benefit of the patients that there are a variety of insulin preparations available, since no single preparation will be the best insulin for all patients.

\section{Insulin needs after CD3-antibody therapy in new-onset type 1 diabetes}

Keymeulen B, Vandemeulebroucke E, Ziegler AG, Mathieu C, Kaufman L, Hale G, Gorus F, Goldman M, Walter M, Candon S, Schandene L, Crenier L, De Block C, Seigneurin JM, De Pauw P, Pierard D, Weets I, Rebello P, Bird P,

Berrie E, Frewin M, Waldmann H, Bach JF, Pipeleers D, Chatenoud L

Academic Hospital and Diabetes Research Center, Brussels Free University-VUB, Brussels, Belgium

bart.keymeulen@az.vub.ac.be

N Engl J Med 2005;352:2598-2608

Background: The T-cell-mediated autoimmune pathogenesis of type 1 diabetes leads to loss of insulinsecreting $\beta$ cells. Efforts to prevent the disease progression have targeted $\mathrm{T}$ lymphocytes first by cyclosporine, and later by monoclonal antibodies against CD3. An open study using a humanized antiCD3 antibody hOKT3 $\gamma 1$ (Ala-Ala) in 12 patients with newly-onset type 1 diabetes was promising, since these patients had better $\beta$-cell function after 1 year than the 12 patients who did not receive the treatment with anti-CD3. To provide evidence for this therapeutic principle, a placebo-controlled, randomized multicenter study was performed with an aglycosylated human IgG1 antibody against CD3 (ChAglyCD3).

Methods: A total of 80 patients with newly-diagnosed type 1 diabetes and aged 12-39 years were randomized to receive either ChAglyCD3 or placebo with intravenous infusions over $2-4 \mathrm{~h}$ for 6 consecutive days. Over the 18-month follow-up, residual $\beta$-cell function was assessed with glucose-clamp-induced C-peptide release before and after administration of glucagon. Daily insulin requirement and glycosylated hemoglobin levels were also recorded, and immunologic parameters followed.

Results: At 6,12 and 18 months the patients treated with ChAglyCD3 had better residual $\beta$-cell function both in the absence and presence of glucagon as compared with the subjects in the placebo group. This effect of ChAglyCD3 was most pronounced in persons with higher initial residual $\beta$-cell function at or above the 50th percentile of the 80 patients, and these patients also had lower daily insulin dose at 18 months $(0.22$ vs. $0.61 \mathrm{IU} / \mathrm{kg} ; \mathrm{p}<0.001)$. In this subgroup, 12 of 16 patients $(75 \%)$ had a daily insulin dose $<0.25 \mathrm{IU} / \mathrm{kg}$ as compared with none in the placebo group. Administration of ChAglyCD3 was associated with transient fever, headache, gastrointestinal symptoms, arthralgia and myalgia. Transient symptoms of Epstein-Barr virus mononucleosis were seen in $75 \%$ of the patients who received ChAglyCD3.

Conclusion: Preservation of residual $\beta$-cell function for at least 18 months was obtained by a short-course treatment with CD3 antibody in subjects with recent-onset type 1 diabetes.

This well-designed and carefully performed multicenter study reports important data on the use of humanized antibody against CD3 in the preservation of residual $\beta$-cell function in subjects with newly diagnosed type 1 diabetes. Endogenous insulin secretion was measured at 6-month intervals in a sophisticated way during prolonged glucose stimulation first in the absence of exogenous glucagon stimulation and next in the presence of this stimulus. The beneficial effect of ChAglyCD3 was clearly seen both in the absence and presence of glucagon. Particularly patients with higher initial residual $\beta$-cell function showed markedly better endogenous insulin secretion together with low daily insulin doses and significantly lower glycosylated hemoglobin levels, too. These observations indicate that treatment with ChAglyCD3 is more beneficial when given at an earlier stage of the disease, which is logical, when remembering that the destruction of $\beta$ cells starts several months or 
years before the clinical diagnosis of diabetes. As the authors point out, the therapeutic potential of ChAglyCD3 will depend on its safety. All 40 patients who received ChAglyCD3 had transient adverse effects during the administration, and $75 \%$ had a clinical syndrome with symptoms similar to mononucleosis (sore throat, fever, and/or cervical adenopathy) within the next 2-4 weeks. These patients also had a transient increase in EBV DNA copies in quantitative PCR analysis from peripheral white cells, followed by an increased proportion of EBV-specific CD8 ${ }^{+} \mathrm{T}$ cells as well as increased titers of IgM and IgG EBV antibodies. Although the levels of EBV-specific DNA returned to baseline, and the pattern of events clearly is different than the reactivation of EBV seen in patients with chronic immunosuppression, potentially leading to lymphoproliferative disease, we have to ask whether we accept an adverse event such as this for children with newly diagnosed type 1 diabetes in the hope that their residual $\beta$-cell function may be better preserved during the first 18 months? It may also be possible that several courses of treatment with ChAglyCD3 are needed, and in that case the issue is whether we would see EBV activation on several occasions in such patients? One issue to remember is that the mechanisms how ChAglyCD3 actually works are currently not known, and therefore the safety issues definitely need to be further studied, and the patients of the present cohort should be observed further. Investigators using another form of humanized, non-mitogenic CD3 antibody, hOKT3y1(Ala-Ala), recently published results extending up to 2 years from the diagnosis of type 1 diabetes and the treatment with hOKT3 $\gamma 1$ (Ala-Ala) [14]. Improved C-peptide production to a mixed meal were observed after 1 and 2 years, and increased relative numbers of CD8 ${ }^{+} \mathrm{T}$ cells after the treatment predicted a favorable clinical response. In conclusion, treatment with CD3 antibody looks promising in preserving $\beta$-cell function, and if shown to be safe, may result in an adjunct therapy in type 1 diabetes, and it may also be considered in the prevention of type 1 diabetes in subjects with signs of preclinical disease.

\title{
A randomized controlled trial of insulin pump therapy in young children with type 1 diabetes
}

\author{
Fox LA, Buckloh LM, Smith SD, Wysocki T, Mauras N \\ Nemours Children's Clinic, NE Florida Pediatric Diabetes Center, Jacksonville, Fla., USA \\ Ifox@Iwpes.org
}

Diabetes Care 2005;28:1277-1281

Background: Insulin treatment is challenging in young children and toddlers with type 1 diabetes. Insulin pump therapy (continuous subcutaneous insulin infusion, CSII) could offer a preferential way to provide insulin therapy than several daily injections. This study was designed to determine whether the use of CSII has a beneficial effect on metabolic control of diabetes, frequency of hypoglycemia, and family life when compared to current therapy with insulin injections in preschool-aged children.

Methods: Twenty-six children being 1-6 years of age and having type 1 diabetes for at least 6 months were randomized to CSII or current therapy (two or three injections of NPH insulin and rapid-acting analog) for 6 months. After 6 months' current therapy, subjects were offered CSII. Hemoglobin A1C (HbA1c) measured by Bayer DCA $2000+$, mean blood glucose (MBG) and hypoglycemia frequency were recorded at 0,1,3 and 6 months. Diabetes-related quality of life (QOL) and parental adjustment were evaluated with questionnaires at baseline and 6 months.

Results: Eleven subjects from each group (84.6\%) completed the trial. Mean HbA1c values were slightly lower but not statistically different between CSII and current therapy groups at baseline (7.43 vs. 7.57), 3 months (7.20 vs. 7.46$)$ and 6 months (7.24 vs. 7.46). MBG was similar between the groups at all time points. Subjects on CSII had higher frequency of fasting mild/moderate hypoglycemia at 1 and 6 months, and predinner mild/moderate hypoglycemia at 3 and 6 months. The frequency of severe hypoglycemia (blood glucose level $<2.2 \mathrm{mmol} / \mathrm{l}$ ), ketoacidosis or hospitalization was similar between the groups. Diabetes-related QOL improved in CSII fathers and psychological distress increased in current therapy mothers from baseline to 6 months. All subjects chose to continue with CSII after the study.

Conclusion: CSII is safe and as effective as injection therapy in maintaining good metabolic control in toddlers and young children with diabetes. CSII may have positive effects on QOL. Mild/moderate hypoglycemia is more frequent with CSII, but the frequency of severe hypoglycemia is not increased. The benefits and realistic expectations of CSII should be thoroughly discussed before starting this therapy in very young children. 
Treatment of diabetes in preschool-aged children is recognized to be challenging. Pain and fear may be associated with injections making everyday life difficult. Variable appetite and eating patterns as well as quickly changing physical activity typically result in widely fluctuating blood glucose values. Young children may be very sensitive to small amounts of insulin. On the other hand, these children frequently experience acute infections and often also need higher insulin doses. The parents may feel increased stress, particularly fear of hypoglycemia, when treating the child with insulin. Insulin pump therapy could offer an easier and more precise way of insulin administration in very young children. Only a few previous studies have evaluated insulin pump treatment in young children, the number of patients being small in all the studies published so far. This study, although also with a small sample size $(n=26)$ but with the advantage of a randomized controlled design, adds a piece of evidence to our knowledge when considering insulin pump treatment in preschool-aged children. Continuous subcutaneous insulin infusion (CSII) did not result in improved diabetes control, maybe due to the fact that the patients already had low $\mathrm{HbA1c}$ levels at baseline. However, $\mathrm{HbA} 1 \mathrm{C}$ values were continuously slightly lower in the CSII group, and probably this difference would have been statistically significant if the sample size had been larger. Whether the risk of mild/moderate or even severe hypoglycemia really is higher on CSII and whether there is a consistent improvement in QOL in the CSII families remain to be confirmed in large randomized controlled trials, which need to be performed in the near future. If CSII were clearly superior to injection therapy in very young patients the next issue would be to find the time and resources needed to train and support the families during the first months of CSII treatment.

\section{Use of multisystemic therapy to improve regimen adherence among adolescents with type 1 diabetes in chronic poor metabolic control: a randomized controlled trial}

Ellis DA, Frey MA, Naar-King S, Templin T, Cunningham P, Cakan N

Department of Psychiatry and Behavioral Science, Wayne State University, Detroit, Mich., USA

dellis@med.wayne.edu

Diabetes Care 2005;28:1604-1610

Background: This study aimed at assessing whether multisystemic therapy (MST), an intensive, homebased psychotherapy, could improve adherence and metabolic control and decrease rates of hospitalization among adolescents with chronically poorly controlled type 1 diabetes.

Methods: 127 American adolescents with type 1 diabetes and chronically poor metabolic control (HbA1c $\geq 8 \%$ for the past year) were randomly assigned to MST and received treatment for about 6 months. Data were collected at baseline and 7 months after the treatment was completed. Changes in A1C adherence, as measured by semistructured interviews and blood glucose meters and hospital admissions and emergency department visits, were assessed.

Results: Significant improvements were seen in the frequency of blood glucose testing as assessed by blood glucose meter readings $(p=0.001)$ and 24 -hour recall interviews $(p=0.011)$ among the patients participating in MST in intent-to-treat analyses. MST participants also had a decreasing number of inpatient admissions, while control subjects had an increased number of inpatient admissions $(\mathrm{p}=0.014)$. Per protocol analyses resulted in similar outcomes but showed in addition a significant improvement in metabolic control for adolescents participating in MST compared with control subjects $(\mathrm{p}=0.047)$.

Conclusions: Adolescents with chronically poorly controlled type 1 diabetes benefit from intensive, homebased psychotherapy in terms of increased blood glucose testing, improved metabolic control, and a decreased hospitalization rate.

Adolescents with type 1 diabetes and long-term poor metabolic control are a challenge to most teams taking care of young patients with diabetes. They may be a source of headache and frustration for all team members, since in spite of considerable efforts there is often hardly any progress in terms of improved glycemic control. This study presents data indicating that a home-based, family-centered, community-based intervention can result in improved metabolic control, improved treatment adherence, and a decreased hospitalization rate in this difficult-to-treat population. The therapy focused on adherence-related problems within the family system, peer network, and the broader community system and used a menu of cognitive-behavioral therapy, parent training and behavioral family systems therapy. One of the strengths of this study is that it was implemented as a randomized 
controlled trial, in which the control group received standard care. The intervention is definitely intensive and costly, and it would be important to assess the cost-effectiveness of this treatment approach. The decreased hospitalization rate results directly in reduced costs, and in addition the improvement achieved in metabolic control would, if sustained, decrease the risk of long-term complications. The present cohort should be observed for a longer period of time to assess whether the achieved improvements are persistent or not. In any case, this study illustrates that successful intervention in diabetic adolescents with chronically poor metabolic control requires a 'holistic' strategy targeting both the family and the immediate environment in addition to the adolescent him/herself.

\section{New mechanisms}

\section{Efficient delivery of siRNA into cytokine-stimulated insulinoma cells silences Fas expression and inhibits Fas-mediated apoptosis}

Burkhardt BR, Lyle R, Qian K, Arnold AS, Cheng H, Atkinson MA, Zhang YC

Department of Pathology, Children's Hospital of Philadelphia, University of Pennsylvania, Pa., USA

czhang@hsc.usf.edu

FEBS Lett 2006;580:553-560

Background: RNA interference (RNAi), via small interfering RNA (siRNA), can silence gene expression in a specific and potent manner in mammalian cells. Since Fas/FasL interactions have been proposed to be important mediators in $\beta$-cell death, this study was designed to investigate the effects of silencing Fas expression with siRNA in mouse insulinoma cells.

Methods: Two mouse insulinoma cell lines were used: NIT-1 derived from non-obese diabetic (NOD) mice and $\beta$ TC-3 derived from C57BL/6 mice. Three siRNA sequences (one control siRNA) were designed based on murine Fas mRNA sequence. Treatment of the cells with medium, siRNA or control siRNA for 3 or 9 days preceded the stimulation of the cells with proinflammatory cytokines IL-1 $\beta$ and IFN- $\gamma$ which induce Fas expression. The magnitude of Fas expression was measured by extracting the total RNA from the cells and performing semiquantitative and real-time reverse transcriptionpolymerase chain reaction (RT-PCR) with specific primers for Fas mRNA. Flow cytometric analysis was used for the detection of cell surface Fas protein and the total Fas protein as well as caspase-3 content in the cells.

Results: In both cell lines cytokine exposure markedly increased Fas mRNA levels, and this induction was efficiently inhibited by treatment with Fas siRNA. Cytokines also induced a rapid elevation of Fas on the cell membrane, which was significantly decreased by Fas siRNA. The total Fas protein in the cells was completely suppressed only after prolonged incubation with siRNA ( 9 days) suggesting a slow turnover of the Fas protein. Furthermore, siRNA significantly inhibited Fas-mediated $\beta$-cell apoptosis assessed by caspase- 3 content of the cells and TUNEL assay, and the extent of apoptosis correlated directly with the level of cell surface Fas.

Conclusion: These results provide additional evidence for the role of the Fas-mediated pathway in $\beta$-cell destruction, and suggest that silencing of Fas with siRNA may be of therapeutic value in prevention of type 1 diabetes and improving cell viability in islet transplantation.

This is a fascinating paper which demonstrates the potential of $\beta$-cell protection with a new technique of specific and efficient gene silencing with siRNA. In this case the total and cell surface expression of Fas was silenced in the insulinoma cells. This resulted in inhibition of the whole apoptotic pathway that is also believed to be involved in $\beta$-cell destruction and development of type 1 diabetes. It must be remembered, however, that this was an in vitro experiment, and multiple mechanisms are likely to mediate $\beta$-cell death in vivo. Keeping this in mind the authors of this paper represent their plans to extend investigations with siRNA to target also other molecules such as iNOS, perforin and cytokine receptors in the hope to protect $\beta$ cells from immune destruction. In addition, they aim at improving islet transplantation by delivering Fas siRNA into primary islets prior to transplantation. These lines of research will be followed with great curiosity in the future, although one has to keep in mind that the role of Fas in $\beta$-cell destruction remains controversial. Pakala et al. [15] 
showed already in 1999 that Fas is not essential for $\beta$-cell destruction in autoimmune diabetes, whereas the transition from benign to destructive insulitis requires an islet cell response to tumor necrosis factor $\alpha$.

\section{The effect of HLA class II, insulin and CTLA4 gene regions on the development of humoral $\beta$-cell autoimmunity}

Hermann R, Laine AP, Lähde J, Veijola R, Simell O, Knip M, llonen J

JDRF Centre for Prevention of Type 1 Diabetes in Finland, Turku, Finland

robert.hermann@utu.fi

Diabetologia 2005;48:1766-1775

Background: $\beta$-Cell autoimmunity precedes the clinical presentation of type 1 diabetes, which is a multifactorial disease associated with a strong genetic predisposition. This study set out to explore the contribution of genetic factors to the emergence of $\beta$-cell-specific humoral autoimmunity.

Methods: The effect of HLA class II, insulin (INS; $-23 \mathrm{HphI}$ variant) and cytotoxic T-lymphocyte-associated protein 4 (CTLA4 [+49 and CT60]) genes on the appearance of $\beta$-cell-specific autoantibodies was analyzed in a large population-based Finnish birth cohort. Infants carrying $H L A-D Q B 1$ genotypes conferring increased risk were monitored for the appearance of autoantibodies (islet cell autoantibodies [ICA], insulin autoantibodies [IAA], glutamic acid decarboxylase autoantibodies [GADA] and islet antigen 2 antibodies [IA-2A]). The study population comprised those who developed $\beta$-cell-specific autoantibodies ( $\mathrm{n}=574$, mean follow-up time 4.9 years; range $0.5-9.3$ ).

Results: Children with the $-23 \mathrm{HphI}$ AA INS genotype were characterized by a higher rate of the emergence of IAA than those carrying AT or TT variants (hazard ratio 2.1, 95\% CI 1.4-2.9; p < 0.001). This effect of the INS locus was present in both those with the HLA-DQB1 high-risk genotype and in those carrying moderate-risk genotypes. There was also a strong association between the appearance of IAA and the HLA-DRB1*0401 allele (hazard ratio 13.1, 95\% CI 1.8-93.4; $\mathrm{p}<0.001$ ). The DRB1*0401 variant accelerated to some extent also the development of IA- $2 \mathrm{~A}(\mathrm{p}=0.03)$. There was no association between isolated ICA positivity and the HLA and INS genotypes. None of the autoantibodies showed any association with the CTLA4 gene.

Conclusions: One of the mechanisms by which the INS and the DRB1 loci confer susceptibility to type 1 diabetes appears to be related the initiation and development of insulin-specific autoimmunity. The emergence of IAA represents a critical step in the ontogeny of $\beta$-cell autoimmunity in young children, in whom the appearance of the other molecular antibodies is linked to IAA.

IAA are usually the first or among the first indicators of $\beta$-cell autoimmunity to appear in young children, who seroconvert to autoantibody positivity when observed prospectively from birth. This raises the issue whether insulin is the primary antigen in the diabetic disease process. This study shows that the development of humoral autoimmunity to insulin is at least partly genetically regulated. The insulin gene polymorphism predisposing to type 1 diabetes was associated with a higher cumulative incidence of IAA over the first 5 years of age in this cohort comprising children with HLA-conferred susceptibility to type 1 diabetes recruited from the general population. In addition, those with the predisposing insulin gene polymorphism had higher IAA levels. A recent study in children with type 1 diabetes demonstrated that those with the predisposing insulin gene polymorphism had higher insulin antibody levels 1 and 6 months after the diagnosis [16]. That observation supports the idea that the predisposing insulin gene polymorphism is associated with an increased immune reactivity to insulin and is in line with the hypothesis that the insulin gene-conferred disease susceptibility is mediated by decreased expression of insulin in fetal thymus resulting in the escape of insulin-reactive $T$ cells into the periphery. In a follow-up study to the present one, we have found that the diseasepredisposing polymorphism of the PTPN22 gene is also related to an increased cumulative incidence of IAA and to rapid progression to overt type 1 diabetes [17]. This emphasizes the critical role of insulin autoimmunity in the development of type 1 diabetes. 


\section{Autoimmune-associated lymphoid tyrosine phosphatase is a gain-of-function variant}

Vang T, Congia M, Macis MD, Musumeci L, Orru V, Zavattari P, Nika K, Tautz L, Tasken K, Cucca F, Mustelin T, Bottini N

Program on Inflammatory Disease Research, Infectious and Inflammatory Disease Center, The Burnham Institute, La Jolla, Calif., USA

nunzio@usc.edu

Nat Genet 2005;37:1317-1319

Background: The SNP 1858C $\rightarrow$ T in the PTPN22 gene encoding lymphoid tyrosine phosphatase (LYP) has been confirmed to be associated with type 1 diabetes, rheumatoid arthritis, Graves disease and other autoimmune diseases. This SNP results in an amino acid change at position 620 in the tyrosine phosphatase protein, the disease-predisposing variant being LYP-Trp620. These investigators hypothesized that altered T-lymphocyte function could be the reason for increased susceptibility to autoimmune disease in patients carrying LYP-Trp620 as compared with the normal LYP-Arg620 variant.

Methods: $\mathrm{T}$ lymphocytes were isolated from individuals with type 1 diabetes with and without the disease-predisposing PTPN22 allele ( $\mathrm{n}=5$ and $\mathrm{n}=4$, respectively). After $20 \mathrm{~h}$ stimulation with anti-CD3 and anti-CD28, phorbol ester plus ionomycin (positive control) and pure medium, interleukin-2 (IL-2) concentrations in the supernatant were measured by ELISA. Primary human T lymphocytes and Jurkat T cells were transfected with LYP-Trp620 or LYP-Arg620 variants either alone or together with a luciferase reporter gene driven by the nuclear factor of stimulated T cells (NFAT)/activator protein-1 (AP-1) transcription factor complex. After activation, IL-2 production and luciferase activity were measured. In addition, the effects of both variants on phosphorylation of T-cell receptors (TCR) and TCRinduced calcium mobilization were studied in primary T cells. The catalytic activities of LYP-Trp620 and LYP-Arg620 proteins immunoprecipitated from the cells were determined.

Results: T cells from carriers of the predisposing PTPN22 $1858 \mathrm{~T}$ allele produced significantly less IL-2 upon TCR stimulation than did T cells from individuals without this allele. In transfected cells, the disease-associated LYP-Trp620 variant consistently inhibited the TCR-induced IL-2 response to a higher extent. Both LYP variants inhibited the TCR-mediated stimulation in a dose-dependent manner when measured by luciferase activity after various doses of plasmids in the transfection were used, but the dose-response curve of LYP-Trp620 was shifted to the left relative to LYP-Arg620, again suggesting that LYP-Trp620 is a more efficient negative regulator of T-cell activation than LYP-Arg620. Phosphorylation of proteins and TCR-induced calcium mobilization were lower in cells expressing LYP-Trp620. The catalytic activity of LYP-Trp620 was $57.2 \%$ higher than that of LYP-Arg620.

Conclusion: The PTPN22 variant encoding LYP-Trp620 and predisposing to autoimmune diseases is a gain-of-function form of the enzyme.

This paper provides very interesting data on the functional mechanisms which could explain the increased susceptibility to autoimmune diseases in subjects with the disease-associated PTPN22 allele. The study shows in several different experiments, all pointing to the same conclusion, that the riskassociated LYP Trp620 variant results in gain of PTPN22 phosphatase activity in human T cells. It still seems, however, that the effects of the LYP-Trp620 variant on the human immune system are rather complicated and cannot be completely understood. How is the increased activity of the LYP-Trp620 variant, which results in more efficient dephosphorylation of proteins, linked to the pathogenesis of human autoimmunity? At which level is the enhanced activity of this enzyme critical; during the thymic process of T-cell selection or in the regulation of peripheral regulatory T cells? An editorial in the same issue of Nature Genetics addresses these questions [18]. The editorial refers to earlier studies on the PEP knockout mice (mouse ortholog of PTPN22) showing that lack of this enzyme causes overactive immunity together with changes in the phosphorylation state of protein tyrosine kinases that regulate proximal events in TCR signaling. Although these mice do not have autoimmunity, it has been hypothesized that in man the observed association between the Trp620 variant and autoimmune diseases would also be explained by a reduction in the activity of the PTPN22 enzyme, which could result in hyperactivity of autoreactive $T$ cells. Therefore, the results of the present paper are surprising, indicating that the gain of function, not loss of function, of PTPN22 is a risk factor for 
autoimmunity. Further studies are needed to entirely understand the association between this phosphatase and autoimmune diseases. It is fascinating that the PTPN11 mutations causing Noonan syndrome also confer gain of function [19].

\section{References}

1. Kim CY, Quarsten H, Berseng E, Khosla C, Sollid LM: Structural basis for HLA-DQ2-mediated presentation of gluten epitopes in celiac disease. Proc Natl Acad Sci USA 2004;101:4175-4179.

2. Knip M, Veijola R, Virtanen SM, Hyöty H, Vaarala O, Åkerblom HK: Environmental triggers and determinants of $\beta$-cell autoimmunity and type 1 diabetes. Diabetes 2005;54(suppl 2):S125-S136.

3. Kent SC, Chen Y, Bregoli L, Clemmings SM, Kenyon NS, Ricordi C, Hering BJ, Hafler DA: Expanded T cells from pancreatic lymph nodes of type 1 diabetic subjects recognize an insulin epitope. Nature 2005;435:224-228.

4. Fourlanos S, Narendran P, Byrnes GB, Colman PG, Harrison LC: Insulin resistance is a risk factor for progression to type 1 diabetes. Diabetologia 2004;47:1661-1667.

5. Mrena S, Virtanen S, Laippala P, Kulmala P, Hannila ML, Åkerblom HK, et al: Models for predicting type 1 diabetes in siblings of affected children. Diabetes Care 2006;9:662-667.

6. Levy AP, Roguin A, Hochberg I, Herer P, Marsh S, Nakhoul FM, et al: Haptoglobin phenotype and vascular complications in patients with diabetes. N Engl J Med 2000;343:969-970.

7. Laing SP, Jones ME, Swerdlow AJ, Burden AC, Gatling W: Psychosocial and socioeconomic risk factors for premature death in young people with type 1 diabetes. Diabetes Care 2005;28:1618-1623.

8. Strudwick SK, Carne C, Gardiner J, Foster JK, Davis EA, Jones TW: Cognitive functioning in children with early onset type 1 diabetes and severe hypoglycemia. J Pediatr 2005;147:680-685.

9. Suh SW, Aoyama K, Matsumori Y, Liu J, Swanson RA: Pyruvate administered after severe hypoglycemia reduces neuronal death and cognitive impairment. Diabetes 2005;54:1452-1458.

10. Barker JM, Goehrig SH, Barriga K, Hoffman M, Slover R, Eisenbarth GS, et al: Clinical characteristics of children diagnosed with type 1 diabetes through intensive screening and follow-up. Diabetes Care 2004;27:1399-1404.

11. Srikanta S, Ganda OP, Gleason RE, Jackson RA, Soeldner JS, Eisenbarth GS: Pre-type 1 diabetes. Linear loss of $\beta$-cell response to intravenous glucose. Diabetes 1984;33:717-720.

12. Skinner TC, Hoey H, McGee HM, Skovlund SE, Hvidøre Study Group on Childhood Diabetes: A short form of the Diabetes Quality of Life for Youth questionnaire: exploratory and confirmatory analysis in a sample of 2,077 young people with type 1 diabetes mellitus. Diabetologia 2006;49:621-628.

13. Teeter JG, Riese RJ: Dissociation of lung function changes with humoral immunity during inhaled human insulin therapy. Am J Respir Crit Care Med 2006 [published ahead of print on March 23, 2006 as doi:10.1164/rccm.2005121861OC].

14. Herold KC, Gitelman SE, Masharani U, Hagopian W, Bisikirska B, Donaldson D, et al: A single course of anti-CD3 monoclonal antibody hOKT3 $\gamma 1$ (Ala-Ala) results in improvement in C-peptide responses and clinical parameters for at least 2 years after onset of type 1 diabetes. Diabetes 2005;54:1763-1769.

15. Pakala SV, Chivetta M, Kelly CB, Katz JD: In autoimmune diabetes the transition from benign to pernicious insulitis requires an islet cell response to tumor necrosis factor $\alpha$. J Exp Med 1999;189:1053-1062.

16. Nielsen LB, Mortensen HB, Chiarelli F, Holl R, Swift P, de Beaufort C, et al: Impact of IDDM2 on disease pathogenesis and progression in children with newly diagnosed type 1 diabetes: reduced insulin antibody titers and preserved $\beta$-cell function. Diabetologia 2006;49:71-74.

17. Hermann R, Lipponen K, Kiviniemi M, Veijola R, Simell O, Knip M, et al: Lymphoid tyrosine phosphatasel (PTPN22) Arg620Trp variant regulates insulin autoimmunity and progression to type 1 diabetes. Diabetologia 2006;49, in press, [Epub ahead of print].

18. Gregersen PK: Gaining insight into PTPN22 and autoimmunity. Nat Genet 2005;37:1300-1302.

19. Uhlen P, Burch PM, Zito CI, Estrada M, Ehrlich BE, Bennett AM: Gain-of-function/Noonan syndrome SHP-2/Ptpn11 mutants enhance calcium oscillations and impair NFAT signaling. Proc Natl Acad Sci USA 2006;103:2160-2165. 